\title{
Detection of the Viable Myocardium. A Perfusion Scintigraphic Study, Before and After Coronary Bypass Surgery in Myocardial Infarction Patients
}

\author{
William A. Chalela, Paulo J. Moffa, José A. F. Ramires, Aguinaldo P. Moraes, \\ José Soares Jr, José C. Meneghetti
}

São Paulo, SP - Brazil

\begin{abstract}
Objective - To compare single-photon-emission computed tomography (SPECT) imaging scans using ${ }^{201}$ Tl and ${ }^{99 m} T c-M I B I$ in detection of viable myocardium, in regions compromised by infarction.
\end{abstract}

Methods - Thirty-two (59.3 \pm 9.8 years old and $87 \%$ male) myocardial infarction patients were studied. All had $Q$ waves on the ECG and left ventricle ejection fraction of $<50 \%$. They underwent coronary and left ventricle angiographies and SPECT before (including ${ }^{201}$ Tl reinjection) and after coronary artery bypass surgery $(C A B G)$. Improvement in perfusion observed after surgery was considered the gold standard for myocardial viability.

Results - Among 102 studied regions of the heart, there were $40(39.2 \%)$ areas of transient perfusion defects in the conventional protocol with ${ }^{201} \mathrm{Tl}$ and $52(51.0 \%)$ after reinjection. Therefore, 12/62 (19.4\%) more viable regions were identified by reinjection. Using ${ }^{99 m} T c-M I B I$, only 14 (13.7\%) regions with transient defects were identified, all of which were seen also in ${ }^{201}$ Tl protocols. After surgery, 49 of a total of 93 regions analyzed (52.7\%) were viable. Sensitivity, specificity, accuracy, positive and negative prediction values were, respectively, ${ }^{201}$ TI SPECT scans- $65.3 \%, 90.9 \%, 77.4 \%$, $88.9 \%$ and $70.2 \%$, reinjection protocol with ${ }^{201} \mathrm{Tl}$ scans $81.6 \%, 81.8 \%, 81.7 \%, 83.3 \%$ and $80.0 \%{ }^{99 m}{ }^{96}$-MIBISPECT scans $-20.4 \%, 90.9 \%, 53.8 \%, 71.4 \%$ and $50.6 \%$. Logistic regression demonstrated that the reinjection protocol with ${ }^{201} \mathrm{Tl}$ was the best predictor of viability $(P<0.001)$.

Conclusion - Our data suggest the election of ${ }^{201}$ Tl for viability studies, especially when using the reinjection protocol.

Keywords: myocardial viability, coronary artery disease, myocardial perfusion scintigraphy

Heart Institute, Hospital das Clínicas - FMUSP

Mailing address: William Azem Chalela - Incor - Serviço de Radioisótopos Av. Dr. Enéas C. Aguiar, 44-AB - 05403-000 - São Paulo, SP - Brazil
Ischemic cardiomyopathy is a frequent cause of hospitalization and death. In addition, patients with severe ischemic heart disease have high mortality, when treated medically, with about a $31 \%$ two-year survival rate ${ }^{1}$. Cardiac transplantation is sometimes indicated for these patients ${ }^{2,3}$.

Ischemic cardiomyopathy can be due to ventricular dysfunction caused by large areas of infarction or by hypokinetic regions caused by chronic hypoperfusion without necrosis. The presence of viable myocardium could favor the indication of myocardial revascularization, thus halting the evolution to larger necrotic areas with the consequent ventricular dysfunction and the need for heart transplantation ${ }^{4}$.

The correct identification of the state of myocardial viability constitutes an important element in the choice of the therapy subsequently adopted: myocardial revascularization, resection of aneurysms, or heart transplantation.

Often, clinical data, the electrocardiogram (ECG), coronary angiography and the analysis of regional wall motion at rest, are not enough for the distinction of fibrosis from viable myocardium.

Initially, postextrasystolic potentiation and infusion of inotropic agents were used to identify viable myocardium based upon the observation of the recovery of myocardial contraction using sensors placed directly on the epicardium under experimental conditions, or by ventricular wall motion analysis in conventional ventriculograms. Recently, echocardiography and gated radionuclide angiography with pharmacological stress (low dose dobutamine) were used for the same purpose.

Positron emission tomography is considered the most sensitive noninvasive technique for the identification of viability. It allows the evaluation of regional flow (nitrogen-13 labeled ammonia) and the myocardial carbohydrate metabolism (fluorine-18 labeled glucose) and lipids (carbon-11 labeled palmitate). However, its use is limited due to the high cost of the equipment required, the need for a multiprofessional team, and also to the extremely short half-life of the tracers. 
Cardiac imaging techniques using myocardial perfusion scintigraphy (MPS), based on the study of myocardial perfusion and cellular membrane integrity, have achieved substantial success in the assessment of myocardial viability ${ }^{5}$.

Thus, the present work aimed to investigate the presence of viable muscle in myocardial infarction areas using single-photon-emission computed tomography (SPECT). For that purpose, both thallium-201 chloride $\left({ }^{201} \mathrm{Tl}\right)$ and 2methoxi-isobuthyl-isonitrile labeled with technetium-99m ( $\left.{ }^{99 \mathrm{~m}} \mathrm{Tc}-\mathrm{MIBI}\right)$ were used. Improvement in perfusion of these areas after coronary artery bypass graft surgery (CABG) was used in the statistical calculations.

${ }^{201} \mathrm{Tl}$ is the radionuclide most often used as a myocardial perfusion marker in the assessment of myocardial viability. ${ }^{99 m}$ Tc-MIBI is frequently used for the diagnosis of coronary artery disease; however, there are insufficient data about its utility in the detection of the viable myocardium.

\section{Methods}

Thirty-two patients with a history of MI (of a total of 113 patients referred for viability studies) were selected. The patients fulfilled the following inclusion criteria: MI between 3 and 12 months prior; presence of Q waves $(\leq 40 \mathrm{~ms})$ on the ECG; angina and heart failure functional class ${ }^{6}<$ III; global left ventricle ejection fraction (LVEF) $<50 \%$; no absolute contraindications to the stress testing (ST). Patients were excluded for: clinical instability; the use of thrombolytic agents; coronary bypass surgery during an acute MI; bundle branch block on ECG; uncontrolled hypertension; valve disease, pericardial disease or other cardiac diseases.

All patients underwent cardiac catheterization and both rest and stress ${ }^{201} \mathrm{Tl}$ and ${ }^{99 \mathrm{~m}} \mathrm{Tc}-\mathrm{MIBI}$ scintigraphies, up to six months before CABG. Complete revascularization was confirmed $^{7}$, and the patients repeated the radionuclide evaluation after approximately three months and the catheterization up to six months after the procedure. Thallium and technetium studies were performed a minimum of three and a maximum of seven days apart. Patients were under the same medication and doses during the follow-up.

This study was approved by the scientific committee of the Heart Institute and also by the ethics committee of our hospital. All patients agreed to participate in the study after informed consent.

ECG tracings were conventionally obtained on the same days of the scintigraphies. Myocardial regions affected by MI were classified as: septal, anterior, inferior, dorsal and lateral, according to the literature ${ }^{8}$.

Coronary lesions were classified according to the percentage of obstruction: occluded (100\% of the lumen), severe lesions ( $>90 \%$ and $<100 \%$ ); critical lesion from $70 \%$ to $90 \%$; and noncritical lesions $(<70 \%)$. The presence of collateral circulation $(\mathrm{Cc})$ was assessed as well as its origin and intensity ${ }^{9}$. Obstructed arteries were considered infarctrelated arteries depending on the anatomical location of the affected region, i. e., left anterior descending artery (LAD) for septal, anterior and apical regions; right coronary (RC) for inferior region; and circumflex (Cx) arteries for lateral and dorsal regions. When $\mathrm{RC}$ and $\mathrm{Cx}$ had a similar degree of obstruction, the dominant artery assumed to be responsible for the inferior region was the RC and for the dorsal region the $\mathrm{Cx}$ branch.

Ventricular wall motion analysis was performed using left ventriculography, in right anterior oblique (RAO) projection. Ventricular wall motion abnormalities were classified as follows: hypokinesis - discrete, moderate or accentuated diminished motion; akinesis - total absence of motion; dyskinesis - paradoxal systolic expansion of a given LV segment. Wall motion was graded arbitrarily ranging from zero to five, according to LV motion $(0=$ normal, 1 to $3=$ discrete to accentuated hypokinesis; $4=$ akinesis and $5=$ dyskinesis). A change of at least one point was considered an improvement, when comparing parameters before and after the procedure.

Global LVEF was calculated by Dodge's uniplanar method ${ }^{10}$ modified by Kennedy ${ }^{11}$.

Stress tests were conventionally performed ${ }^{12}$, using the treadmill and the Ellestad's protocol ${ }^{13}$, with a 12-lead simultaneous recording.

The protocol of the ${ }^{201} \mathrm{Tl}$ scintigraphy included three phases: $1^{\text {st }}$, imaging was acquired immediately after radionuclide injection at peak exercise; the dose injected was 111 $\mathrm{MBq}(3 \mathrm{mCi})$ and tomographic images obtained with the patient in the supine position; $2^{\text {nd }}$, after $4 \mathrm{~h}$ the redistribution images, similarly acquired; $3^{\text {rd }}$, reinjection, performed after $24 \mathrm{~h}$, with an additional dose of thallium administered at rest and SPECT images acquired after $4 \mathrm{~h}$.

For ${ }^{99 \mathrm{~m}}$ Tc-MIBI studies, the entire procedure was performed in one day. Initially, images were obtained at rest, with a 296 to $370 \mathrm{MBq}$ ( 8 to $10 \mathrm{mCi}$ ) intravenous infusion, followed by the stress images. The radionuclide [ 888 to 1110 $\mathrm{MBq}(24$ to $30 \mathrm{mCi})]$ was injected at peak exercise, three times the resting dose. To acquire tomographic images, a Siemens scintigraphic camera, model OrbiterZLC-Digitrac 750, coupled to a Maxdelta (Microvax - 3300) computer was used. Analog imaging was converted to digital in a $64 \times 64,64$ projections with $20 \mathrm{~s}$ duration each, over a $180^{\circ}$ arch, beginning from $45^{\circ} \mathrm{RAO}$ to $45^{\circ}$ left posterior oblique LPO (step/ shoot acquisition mode). The filter used was a Butterworth, with a cutoff frequency of $0.4 \mathrm{Ny}$ (Ny-Nyquist frequency) for ${ }^{201} \mathrm{Tl}$ and $0.5 \mathrm{Ny}$ for ${ }^{99 \mathrm{~m}} \mathrm{Tc}-\mathrm{MIBI}$.

After image reconstruction, transverse slices every $6.09 \mathrm{~mm}$, along the heart main axis, were obtained allowing the determination of sections corresponding to three plans mutually perpendicular to the coordinate system of the heart: coronal section or horizontal long axis (in the inferoanterior direction); oblique section or short axis (in the apexbase direction); sagittal section or vertical long axis (in the septolateral direction). The heart was divided into five regions: septal, anterior, inferior, apical and lateral (laterodorsal) and, for each area an arbitrary value in a four-point grading system was given according to radionuclide con- 
centration $(0=$ normal perfusion; $1=$ mild perfusion defect, $3=$ severe perfusion defect or tracer absence). A change of at least one point in the grade of a given region was considered as transient perfusion defects and was classified as partial when zero was not observed in the redistribution and/or reinjection images. Maintenance of perfusion values was indicative of persistent perfusion defects (fibrosis).

In this study, we analyzed only LV regions compromised by MI, as shown on the ECG. The apical region was considered as an extension of anterior and/or inferior involvement, when graded with values greater than zero. Areas of improvement in myocardial perfusion observed on the resting images were considered as true viable ones.

To accomplish the goals of this study, descriptive tables were constructed. Continuous variables were presented descriptively as mean and standard deviations. Comparisons before and after surgery were performed with Wilcoxon's (nonparametric) or Student (paired) $t$ tests ${ }^{14}$. Agreement between methods for myocardial viability was analyzed using Kappa statistics ${ }^{15}(\mathrm{~K})$. Values $>0.75$ represent excellent agreement, between 0.40 and 0.75 good agreement, and $<0.40$, poor agreement. Sensitivity $(S)$, specificity
(E), accuracy (A), positive (PPV) and negative (NPV) predictive values were calculated. Viability confirmed after surgery was adjusted using a stepwise logistic regression model in which the other protocols were considered predictive factors.

The significance level used was 0.05. Calculations were made using the SAS system ${ }^{16}$. Intra and interobserver variability analysis was calculated by reevaluating a sample of 10 cases after at least 30 days.

\section{Results}

Studied population data are found in table I. The mean age was $59.3 \pm 9.8$ years (ranging from 42 to 75 years), 28 $(87.6 \%)$ males and $4(12.4 \%)$ females. Patients were studied on average $8.14 \pm 3.29$ months after MI.

Seventy-eight myocardial regions of fibrosis were studied. They were divided into: 23 (29.5\%) septal, 23 $(29.5 \%)$ anterior, $21(26.9 \%)$ inferior, 10 (12.8\%) lateral and 1 $(1.3 \%)$ dorsal regions.

Catheterization (data are displayed in table II) was performed a mean of $2.79 \pm 2.73$ months before surgery. Single vessel disease occurred in one (3.1\%) patient; double

\begin{tabular}{|c|c|c|c|c|c|c|c|c|c|c|c|c|c|c|c|c|c|c|c|c|}
\hline \multirow[t]{2}{*}{ No } & \multirow[t]{2}{*}{ Name } & \multirow{2}{*}{$\begin{array}{c}\text { Age } \\
\text { (years) }\end{array}$} & \multirow{2}{*}{ Gender } & \multirow{2}{*}{$\begin{array}{l}\text { Weight } \\
(\mathrm{kg})\end{array}$} & \multirow{2}{*}{$\begin{array}{l}\text { Height } \\
(\mathrm{Cm})\end{array}$} & \multirow{2}{*}{$\begin{array}{c}\mathrm{BMI} \\
\left(\mathrm{kg} / \mathrm{m}^{2}\right)\end{array}$} & \multirow[t]{2}{*}{ HBP } & \multirow[t]{2}{*}{$\mathrm{DM}$} & \multirow[t]{2}{*}{$\mathrm{HC}$} & \multirow[t]{2}{*}{ HT } & \multirow[t]{2}{*}{$\mathrm{S}$} & \multirow[t]{2}{*}{$\mathrm{FH}$} & \multicolumn{5}{|c|}{ ECG - areas } & \multirow{2}{*}{$\begin{array}{c}\text { Time } \\
\text { (months) }\end{array}$} & \multirow{2}{*}{$\begin{array}{l}\mathrm{FC} \\
\mathrm{AP}\end{array}$} & \multirow{2}{*}{$\begin{array}{l}\text { FC } \\
\text { HF }\end{array}$} \\
\hline & & & & & & & & & & & & & $\mathrm{S}$ & $\mathrm{A}$ & I & $\mathrm{L}$ & $\mathrm{P}$ & & & \\
\hline 1 & GAL & 61 & M & 88 & 1.75 & 28.7 & YES & NO & NO & NO & YES & NO & $\mathrm{S}$ & A & I & & & 3.8 & I & III \\
\hline 2 & CABG & 42 & M & 79 & 1.70 & 27.3 & YES & NO & NO & NO & YES & YES & $\mathrm{S}$ & $\mathrm{A}$ & & $\mathrm{L}$ & & 10.3 & III & III \\
\hline 3 & MIS & 61 & M & 102 & 1.78 & 31.9 & NO & YES & YES & NO & NO & YES & & & I & & & 12.0 & III & III \\
\hline 4 & OVA & 47 & M & 92 & 1.79 & 28.7 & YES & NO & NO & NO & YES & YES & $\mathrm{S}$ & A & I & & & 3.8 & III & II \\
\hline 5 & ACGD & 49 & M & 63 & 1.62 & 24.0 & YES & YES & YES & NO & NO & NO & $\mathrm{S}$ & $\mathrm{A}$ & I & & & 6.8 & II & III \\
\hline 6 & SS & 65 & M & 67 & 1.65 & 24.6 & YES & YES & NO & NO & NO & NO & & & I & $\mathrm{L}$ & & 12.0 & II & II \\
\hline 7 & $\mathrm{TJ}$ & 55 & M & 68 & 1.70 & 23.5 & YES & NO & YES & NO & YES & NO & $\mathrm{S}$ & A & I & & & 4.3 & III & III \\
\hline 8 & $\mathrm{AOA}$ & 74 & M & 63 & 1.72 & 21.3 & NO & NO & NO & NO & YES & NO & $\mathrm{S}$ & A & I & & & 6.9 & III & III \\
\hline 9 & PSS & 72 & M & 86 & 1.72 & 29.1 & YES & YES & NO & NO & YES & YES & $\mathrm{S}$ & $\mathrm{A}$ & & $\mathrm{L}$ & & 11.7 & III & III \\
\hline 10 & PL & 65 & M & 60 & 1.60 & 23.4 & NO & NO & NO & NO & YES & NO & & & I & $\mathrm{L}$ & & 11.7 & I & III \\
\hline 11 & $\mathrm{CP}$ & 48 & M & 86 & 1.74 & 28.4 & NO & NO & NO & NO & YES & YES & $\mathrm{S}$ & A & & $\mathrm{L}$ & & 4.6 & I & II \\
\hline 12 & MTM & 67 & M & 81 & 1.66 & 29.4 & NO & YES & NO & NO & YES & NO & & & I & & & 3.1 & II & II \\
\hline 13 & $\mathrm{OF}$ & 63 & M & 70 & 1.70 & 24.2 & NO & NO & YES & NO & YES & YES & $\mathrm{S}$ & A & & & & 12.0 & III & II \\
\hline 14 & $\mathrm{BM}$ & 72 & M & 65 & 1.80 & 20.1 & NO & YES & NO & NO & YES & NO & $S$ & $\mathrm{~A}$ & & & & 3.3 & II & III \\
\hline 15 & DCA & 63 & M & 68 & 1.73 & 22.7 & NO & YES & NO & NO & NO & NO & $\mathrm{S}$ & $\mathrm{A}$ & I & & & 11.6 & I & III \\
\hline 16 & PAAG & 65 & M & 79 & 1.77 & 25.2 & YES & YES & NO & NO & YES & NO & $S$ & A & & & & 11.1 & I & III \\
\hline 17 & JMP & 54 & $\mathrm{~F}$ & 51 & 1.58 & 20.4 & YES & NO & YES & NO & YES & NO & $S$ & $\mathrm{~A}$ & & & & 12.0 & III & II \\
\hline 18 & $\mathrm{NCP}$ & 65 & $\mathrm{~F}$ & 66 & 1.56 & 27.1 & YES & YES & YES & NO & NO & YES & $S$ & A & I & & & 4.2 & III & II \\
\hline 19 & JBS & 56 & M & 73 & 1.54 & 30.8 & YES & NO & NO & NO & YES & NO & $S$ & A & I & & & 8.7 & I & II \\
\hline 20 & $\mathrm{JMB}$ & 74 & M & 75 & 1.60 & 29.3 & YES & NO & NO & NO & NO & YES & & & I & $\mathrm{L}$ & $\mathrm{P}$ & 11.7 & I & III \\
\hline 21 & $\mathrm{FP}$ & 56 & M & 66 & 1.55 & 27.5 & YES & YES & YES & NO & NO & NO & & & I & $\mathrm{L}$ & & 11.8 & III & I \\
\hline 22 & NGS & 42 & $\mathrm{~F}$ & 52 & 1.46 & 24.4 & NO & YES & YES & NO & NO & NO & $S$ & A & I & & & 5.5 & III & III \\
\hline 23 & $\mathrm{JB}$ & 61 & M & 70 & 1.70 & 24.2 & YES & NO & NO & NO & YES & YES & & & i & & & 11.6 & III & III \\
\hline 24 & GMS & 63 & M & 70 & 1.70 & 24.2 & NO & NO & NO & NO & YES & NO & $\mathrm{S}$ & A & I & $\mathrm{L}$ & & 12.0 & I & III \\
\hline 25 & SAC & 67 & M & 72 & 1.72 & 24.3 & NO & NO & NO & NO & YES & YES & & & I & $\mathrm{L}$ & & 11.1 & III & III \\
\hline 26 & JMC & 52 & M & 85 & 1.73 & 28.4 & YES & YES & NO & NO & YES & YES & S & A & I & & & 12.0 & I & III \\
\hline 27 & SAA & 44 & M & 71 & 1.58 & 28.4 & NO & NO & YES & NO & YES & NO & $\mathrm{S}$ & A & & & & 3.4 & I & III \\
\hline 28 & GDS & 75 & M & 81 & 1.72 & 27.4 & NO & NO & NO & NO & YES & NO & & & I & $\mathrm{L}$ & & 5.7 & II & III \\
\hline 29 & $\mathrm{AM}$ & 65 & M & 82 & 1.71 & 28.0 & YES & YES & YES & NO & NO & NO & $S$ & A & & & & 3.3 & III & III \\
\hline 30 & JCNN & 45 & M & 78 & 1.80 & 24.1 & YES & YES & NO & NO & YES & NO & $S$ & A & I & & & 3.3 & III & III \\
\hline 31 & GTB & 62 & M & 56 & 1.63 & 21.1 & NO & NO & NO & NO & NO & NO & $\mathrm{S}$ & A & & & & 11.9 & III & III \\
\hline 32 & MFL & 49 & $\mathrm{~F}$ & 90 & 1.60 & 35.2 & YES & NO & YES & NO & NO & YES & $S$ & A & I & & & 3.7 & III & III \\
\hline
\end{tabular}




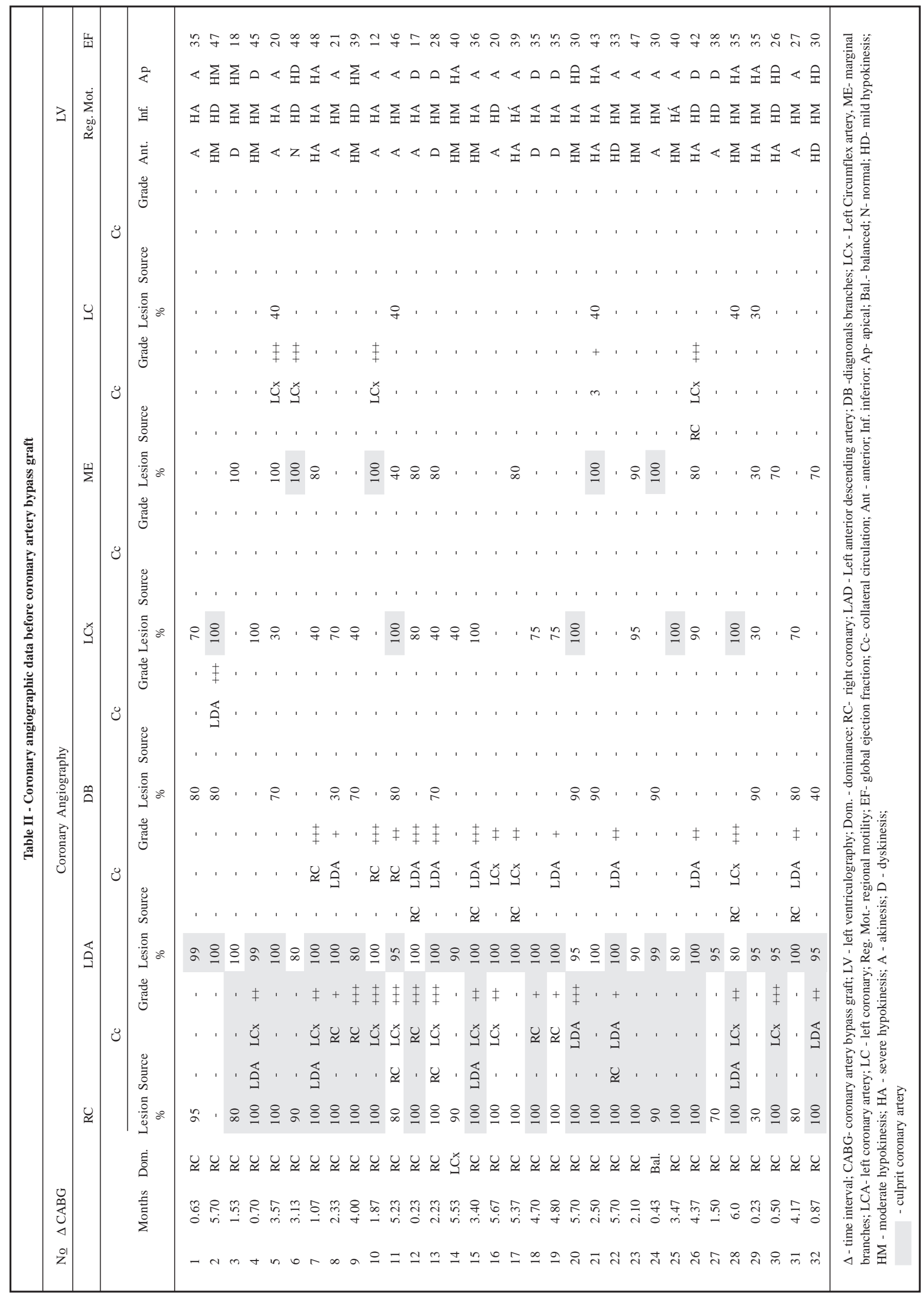


vessel disease in $7(21.9 \%)$, most frequently the LAD and RC combination (71.4\%); triple vessel disease in 24 (75.0\%). Collateral circulation was observed in $24(75.0 \%)$ patients. Regional motion could be studied in the anterior, inferior and apical LV wall, making a total of 96 regions. Changes found were: 61 (63.4\%) regions with hypokinesis, $23(24 \%)$ with akinesis and $11(11.6 \%)$ with dyskinesis. Global LVEF observed in all patients was $34.9 \pm 10.2 \%$. The 3 patients who died in the immediate postoperative (PO) period had LVEF of $20 \%, 30 \%$ and $43 \%$.

There were no significant differences in the main clinical, electrocardiographic, hemodynamic and metabolic stress test variables. Mean maximal heart rates were: $132 \pm 20.6 \mathrm{bpm}$ and $130.5 \pm 18.6 \mathrm{bpm}$; systolic blood pressure, $159.5 \pm 30.5 \mathrm{mmHg}$ and $153.7 \pm 23.2 \mathrm{mmHg}$; and double product $21159.2 \pm 5676.1$ and $20063.0 \pm 4125.9(\mathrm{p}=\mathrm{ns})$. The tracers were injected in similar conditions during both SPECT studies.

Every artery with lesions $\geq 50 \%$ was bypassed. Followup period after MR surgery was $15.16 \pm 10.17$ months. Three $(9.4 \%)$ patients died soon after operation due to cardiogenic shock. Another patient died suddenly after four months. Angina and CHF symptoms were evaluated in twenty-nine $(90.6 \%)$ patients to determine functional class before and after CABG (fig. 1), and statistical analyses showed significant $(\mathrm{p}<0.001)$ improvement in both parameters analyzed.

Coronary angiographic studies after surgery could be performed in 23 patients $(4.98 \pm 1.09$ months postoperatively). All grafts were patent. There was wall motion improvement in $48(69.6 \%)$ regions and no change in $21(30.4 \%)$ myocardial regions, when compared with the preoperative studies ( $\mathrm{p}<0.001)$. Mean global LVEF also increased (46.4 \pm $13.5 \%$; $<<0.001 ;$ fig. 2 ).

For the detection of viable myocardium using scintigraphy, 102 myocardial regions affected by MI were analyzed. ${ }^{201} \mathrm{Tl}$, conventional protocol (comparing stress and $4 \mathrm{~h}$ redistribution imaging) showed $40(39.2 \%)$ reversible regions and $62(60.8 \%)$ nonviable ones (fixed defect); the reinjection protocol (stress and $24 \mathrm{~h}$ reinjection imaging) showed $52(51.0 \%)$ and $50(49 \%)$ myocardial regions to be reversible and irreversible, respectively. Therefore, reinjection identified 12 additional reversible regions (19.4\%) initially classified as irreversible by the conventional study. With the conventional ${ }^{99 \mathrm{~m}} \mathrm{Tc}-\mathrm{MIBI}$ protocol (stress and rest imaging) there were $14(13.7 \%)$ reversible regions and the remaining $88(86.3 \%)$ were considered nonviable (tab. III).

After surgery, 29 of 32 patients were able to undergo scintigraphy, except for the three cases of death in an early postoperative phase. Therefore, 93/102 (91.2\%) regions were analyzed: $21(22.6 \%)$ septal, $21(22.6 \%)$ anterior, 22 (23.7\%) apical, 19 (20.4\%) inferior and 10 (10.7\%) laterodorsal. $49(52.7 \%)$ regions were classified as truly viable, in $41(44.1 \%)$ there was agreement between scintigraphies and

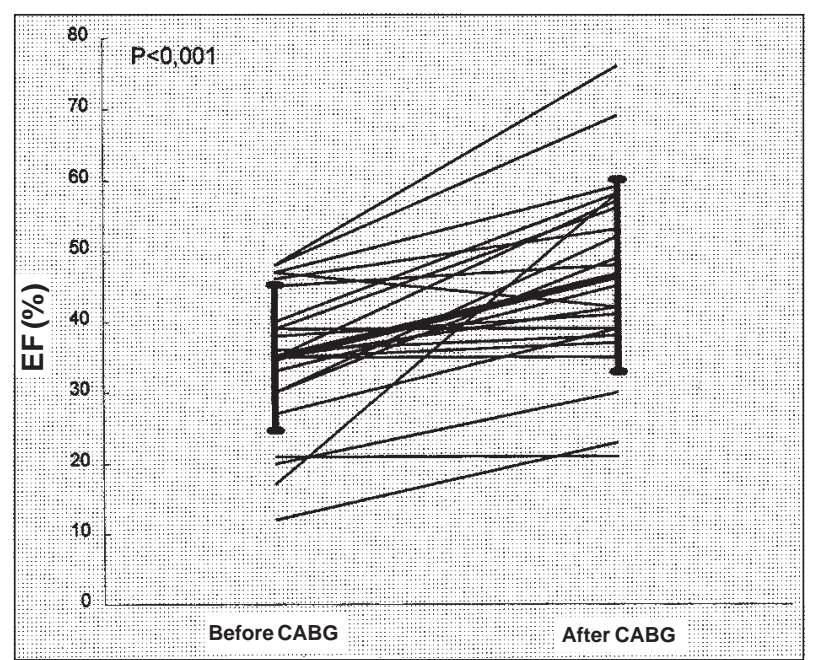

Fig. 2 - Left Ventricle ejection fraction distribution, before and after Coronary Artery Bypass Graft. $\mathrm{N}=23$ patients; $\mathrm{EF}=$ Global Left Ventricle Ejection Fraction; CABG $=$ Coronary Artery Bypass Graft Surgery

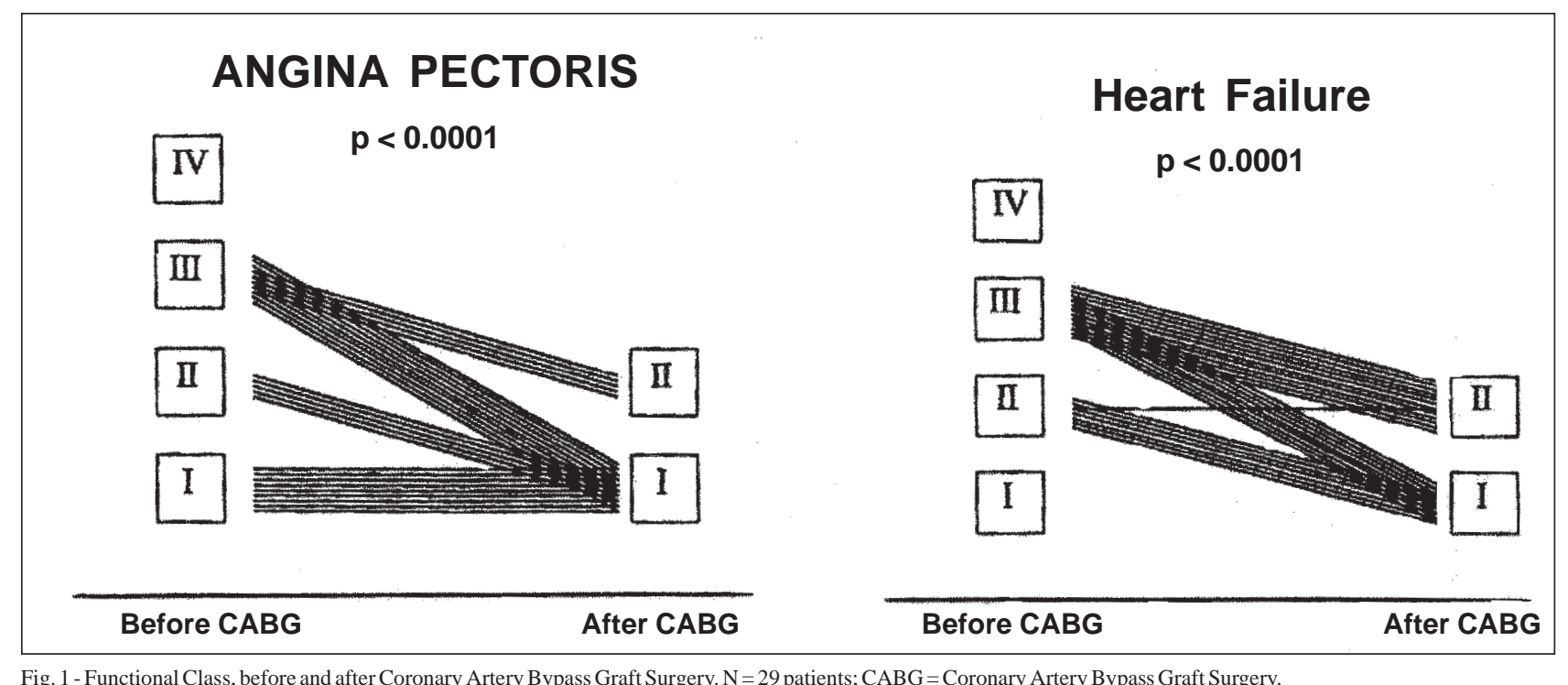




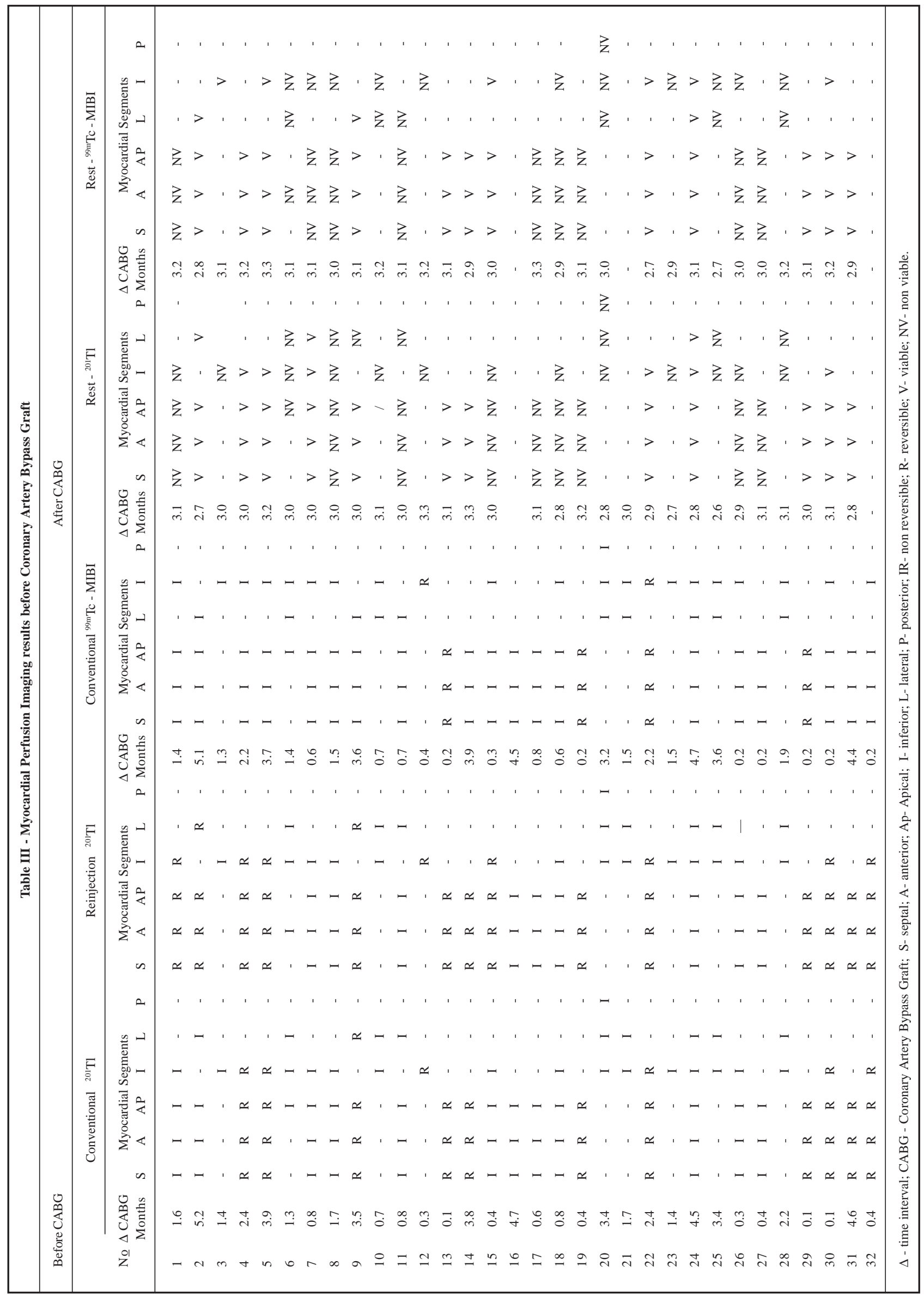


of the remaining $8.4(4.3 \%)$ were viable according to ${ }^{201} \mathrm{Tl}$ and $4(4.3 \%)$ according to ${ }^{99 \mathrm{~m}} \mathrm{Tc}-\mathrm{MIBI}$; the other $44(47.3 \%)$ regions were truly nonviable (tab. III). There was agreement in $85(91.4 \%)$ regions, which is significant $(\mathrm{p}<0.001)$ with excellent reproducibility $(\mathrm{k}=0.828)$.

Figures 3 and 4 show an MPS example with tomographic slices of the oblique axis of a patient with MI and viable muscle.

Calculations of operating variables S, E, A, PPV and NPV in the entire population were, respectively: $65.3 \%$, $90.9 \%, 77.4 \%, 88.9 \%$ and $70.2 \%$ for the conventional proto- col with 201Tl; and $81.6 \%, 81.8 \%, 81.7 \%, 83.3 \%$ and $80.0 \%$ for reinjection one; $20.4 \%, 90.9 \%, 53.8 \%, 71.4 \%$ and $50.6 \%$ for the conventional protocol with $99 \mathrm{mTc}-\mathrm{MIBI}$. It is concluded that there is an underestimation of viable myocardium by the conventional protocol with ${ }^{99 \mathrm{~m}} \mathrm{Tc}-\mathrm{MIBI}$. The increased perfusion, observed after revascularization of myocardial regions with $\mathrm{MI}$ and fibrosis but still viable areas, was shown better by ${ }^{201} \mathrm{Tl}$ SPECT, notably with the reinjection protocol. It could be confirmed, using logistic regression analyses, that the reinjection protocol was the best predictor of viability $(\mathrm{p}<0.001)$.

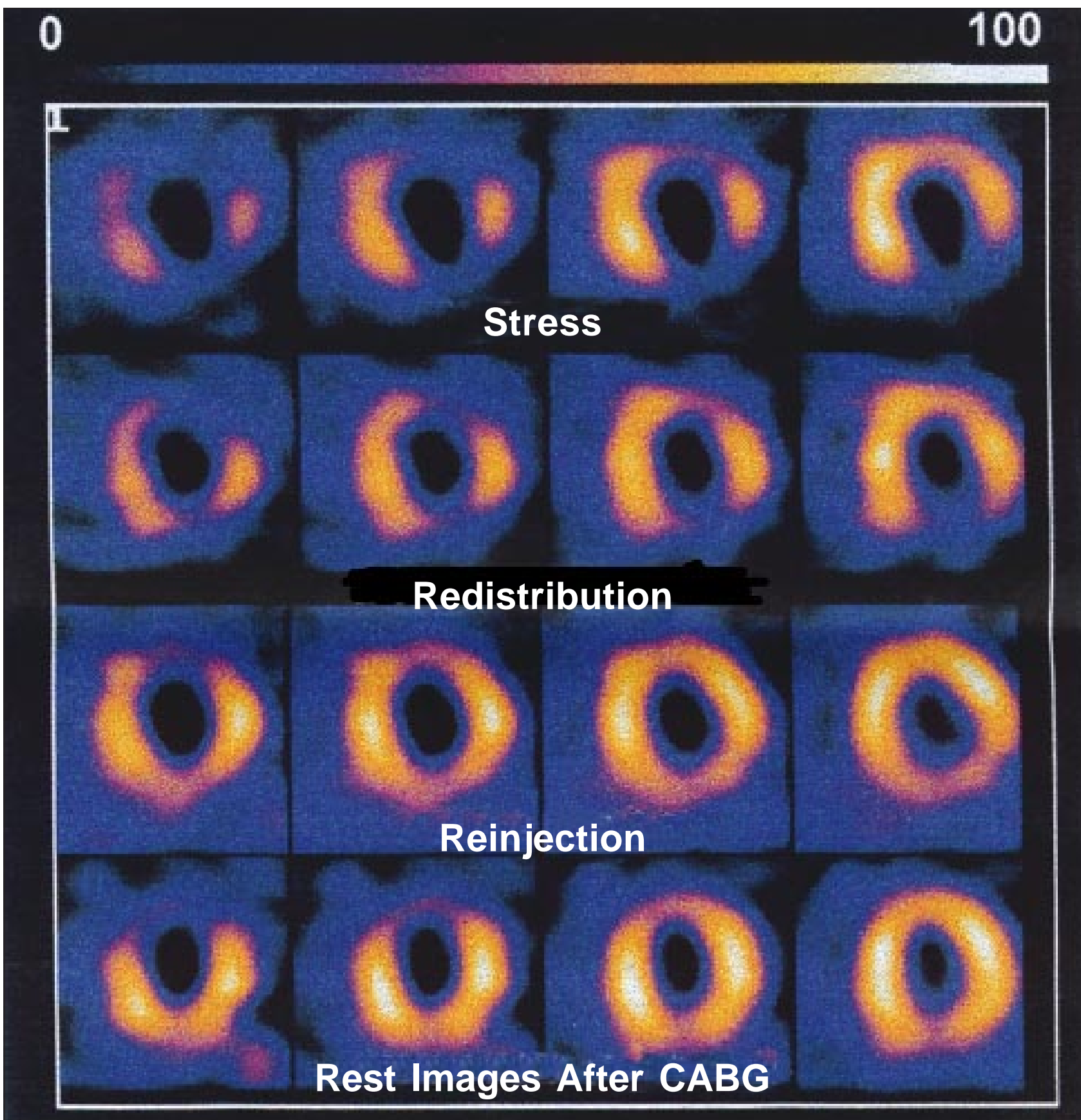

Fig. 3 - Myocardial perfusion scintigraphy with $201 \mathrm{Tl}$ and tomographic slices in the oblique plan. Redistribution images suggest viability in the anterior and lateral regions reinjection suggest viability of the inferior wall. 


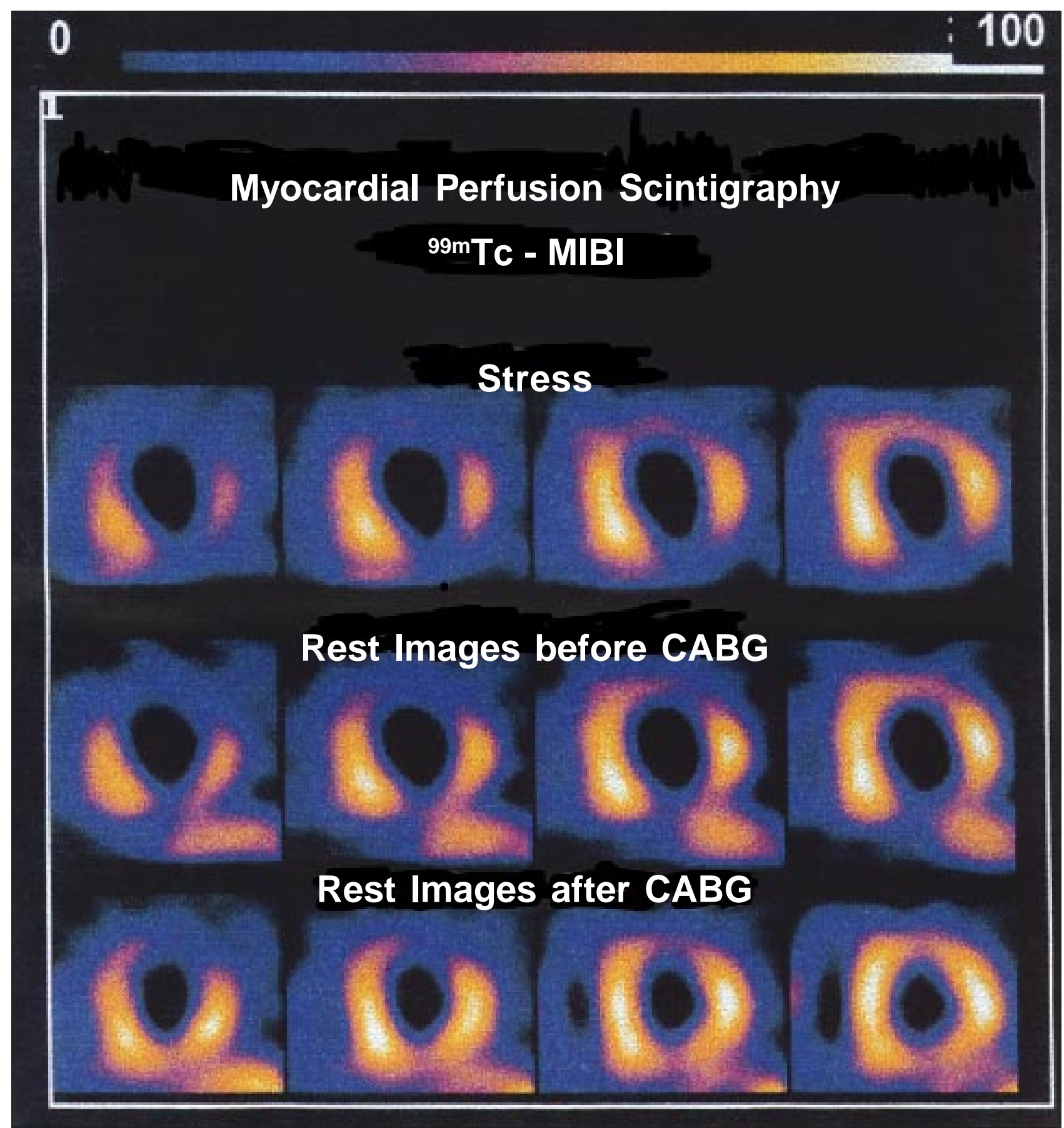

Fig. 4 - Myocardial perfusion scintigraphy with ${ }^{99 \mathrm{~m} T c-M I B I}$ and tomographic slices in the oblique plan. Conventional protocol imaging shows persistent defects in the anterior, inferior and lateral regions. After CABG, images confirmed that these persistent defects contained viable muscle. CABG-Coronary artery bypass graft.

It was observed that, through severe lesions (obstruction $>90 \%$ lumen), coronary and $\mathrm{Cc}$ in the same territory were responsible for perfusion of 24/49 (49.0\%) viable regions and of 26/44 (59.0\%) nonviable ones $(p=n s)$. However, the semiquantified analysis of collateral circulation intensity of $+++/++++$ was more frequent in viable regions $(60 \%)$ and of $+/++$ in nonviable ones $(78.6 \%)$. These differences were significant ( $\mathrm{p}=0.004$, tab. IV).

Forty-nine myocardial regions (of a total of 69) evaluated after revascularization were MI related. There was regio- nal wall motion improvement in 36/49 (73.5\%) after CABG (p<0.001, fig. 5).

The relationship between perfusion and systolic function recovery could be observed in 49 myocardial regions, 34 of which (69.4\%) were in agreement, and 25 (51.\%) improved both perfusion and regional motility, but $9(18.4 \%)$ did not. The results were contradictory in $15(30.6 \%)$ regions. There was an improvement in motion in $11(22.4 \%)$ and in perfusion in 4(8.2\%). However, the improvement in regional perfusion was significantly related $(\mathrm{p}=0.011)$ to the improvement in wall motion. 


\begin{tabular}{|c|c|c|c|c|c|c|}
\hline \multicolumn{7}{|c|}{$\begin{array}{c}\text { Table IV - Relation between the presence and intesity of coronary } \\
\text { collateral circulation and viability on the Myocardial Perfusion } \\
\text { Scintigraphy }\end{array}$} \\
\hline \multirow{3}{*}{$\begin{array}{c}\text { Myocardial } \\
\text { Segments }\end{array}$} & \multicolumn{6}{|c|}{ Collateral Circulation } \\
\hline & \multicolumn{2}{|c|}{$+/++$} & \multicolumn{2}{|c|}{$+++/++++$} & \multirow[b]{2}{*}{$\mathrm{P}$} & \multirow[b]{2}{*}{ Total } \\
\hline & $\mathrm{N}$ & $\%$ & $\mathrm{~N}$ & $\%$ & & \\
\hline Viable & 10 & 40.0 & 15 & 60.0 & \multirow{2}{*}{0.004} & 25 \\
\hline Non Viable & 22 & 78.6 & 6 & 21.4 & & 28 \\
\hline Total & 32 & 60.4 & 21 & 39.6 & & 53 \\
\hline
\end{tabular}

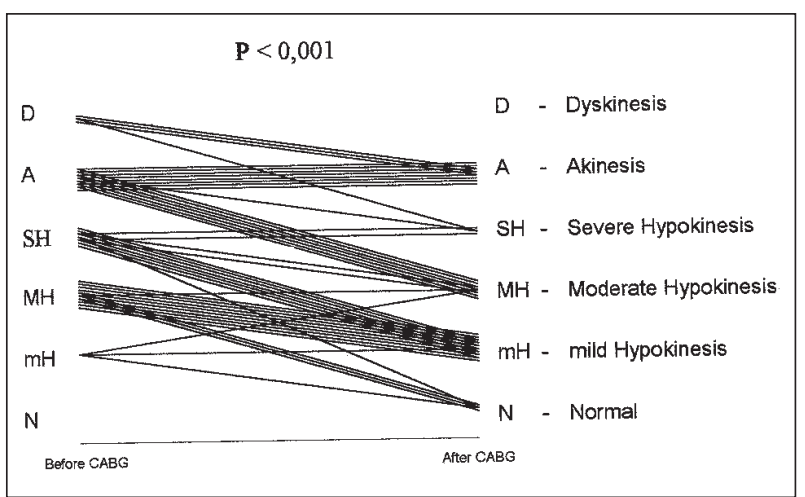

Fig. 5 - Regional motility before and after revascularization of the regions affected by myocardial infarction. D- dyskinesis; A- akinesis; HA- accentuated hypokinesis; HM- moderate hypokinesis; HD- discrete hypokinesis; $\mathrm{N}$ - normal.

\section{Discussion}

In patients with $\mathrm{CAD}$ and $\mathrm{LV}$ dysfunction, $\mathrm{CABG}$ surgery has been shown to have a positive impact on survival. In the present study, survival was $96.6 \%$ during a follow-up period of $15.2 \pm 10.2$ months. Only one of 29 patients discharged $(3.4 \%)$ died suddenly.

The presence of viable myocardium is not the only factor to indicate the need for CABG, but also the size of the area at risk, which once recovered, will lead to changes both in the clinical condition and in the natural history after revascularization. In this sense, surgical benefits could be confirmed by symptoms of angina and heart failure.

The study has also shown an in-hospital survival of 90.6\%. No patient with EF $<20 \%$ died in this phase. 19 patients had $\mathrm{EF} \geq 20 \%$ and $<40 \%$, of whom two died in the immediate postoperative period. Therefore, hospital survival for these patients was $89.5 \%$ (17/19 patients), similar to that observed in the literature. Hochberg et al ${ }^{17}$ studied 466 patients with CAD and LVEF $<40 \%$ who underwent CABG and followed them for 36 months. Hospital survival (up to 30 days after surgery) for patients with $L V E F \geq 20 \%$ and $\leq 30 \%$ was $89 \%$. The CASS study ${ }^{18}$ observed significant surgical benefit in a subgroup of patients with CAD in the three main coronary vessels and LVEF between $35 \%$ and $50 \%$. They also observed increased survival after surgery in patients with LV dysfunction, particularly those with LVEF $\leq 25 \%$, concluding that the presence of severe heart failure does not constitute a specific contra indication for surgery.
These data of increased survival, and the clinical improvement observed, favor the indication for surgery in patients with different degrees of left ventricular dysfunction and CAD. However, the improvement observed after MR represents a complex interaction between compensatory mechanisms, coronary anatomy, surgical results and patient selection.

The later the evaluation - two to three months after surgery - the more correct the interaction between recovery of perfusion and function, perioperative injury and graft patency will be.

In the conventional protocol, there were perfusion scans with ${ }^{201} \mathrm{Tl}$ showing 40/102 (39.2\%) regions of reversibility. After reinjection 52/102 (51.0\%) regions were viable. Therefore, from 62/102 irreversible regions by the conventional protocol, 12/62 (19.4\%) regions were shown to be viable after reinjection. This figure is smaller than that in the literature. Several studies show that the reinjection protocol, as compared with conventional scans with ${ }^{201} \mathrm{Tl}$, lowers the number of defects considered fixed by about 31 to $49 \%{ }^{19-21}$. In the present study, the smaller overestimation of the degree of fibrosis could be justified by the method used, because the redistribution images of the conventional protocol were performed later than in the studies quoted above.

Overestimation of the degree of fibrosis (persistent perfusion defects) by the conventional protocol, when compared with that of ${ }^{201} \mathrm{Tl}$ reinjection, is dependent on the redistribution phenomenon. Soon after the initial ${ }^{201} \mathrm{Tl}$ uptake by the myocardium, there is a continuous exchange of this univalent ion with the blood that determines its redistribution. ${ }^{201} \mathrm{Tl}$ is continuously released from normal myocardium and replaced by recirculating ${ }^{201} \mathrm{Tl}$, from residual activity in the blood. Although its initial biodistribution is similar to $\mathrm{K}+$, compartment redistribution presents a different velocity of exchange between the intra- and extracellular milieu. ${ }^{201} \mathrm{Tl}$ uptake by the cells is dependant on regional availability, as well as on membrane and $\mathrm{Na}+\mathrm{K}+$ pump integrity. However, ischemia-related metabolic changes alter the extraction rate and the exchange with the extracellular medium. In contrast to fibrotic cells, viable myocardium maintains a slow metabolism that preserves the basic cellular structure, making possible the recovery of its original functions. This complex protective mechanism may be detected by nuclear medicine and allows observation, using the dynamic biodistribution of the radionuclide in cardiac muscle. The smaller accumulation and slow washout of ${ }^{201} \mathrm{Tl}$ in the ischemic areas result in the perfusion defects. This process, based upon redistribution, is only possible if myocardium is viable, with an intact cellular membrane ${ }^{22-24}$.

The redistribution phenomenon is, however, highly influenced by serum concentration of the tracer and by cellular washout. Redistribution may occur later in situations in which there is resting low blood flow due to severe coronary stenosis, absence of hyperemia after stress, longlasting metabolic abnormalities and low serum concentration of the tracer ${ }^{22,25}$. Slow redistribution, observed in ischemic (viable) areas, makes the 3 to 4 hour images depen- 
dent on limitations in the differentiation of viable from fibrotic myocardium.

The conventional protocol using ${ }^{99 \mathrm{~m}} \mathrm{Tc}-\mathrm{MIBI}$ showed only 14/102 (13.7\%) viable regions; ${ }^{201} \mathrm{Tl}$ conventional scans identified $26(25.5 \%)$ additional viable regions; and after reinjection, 38 (37.3\%) more regions, when compared with the ${ }^{99 \mathrm{~m} T c-M I B I}$ scans. Therefore, protocols with ${ }^{201} \mathrm{Tl}$ were superior in detecting reversible defects in areas affected by MI. This is similar to findings in the literature.

Cuocolo et $\mathrm{al}^{26}$ studied 20 patients with CAD and LV dysfunction (EF of $30 \% \pm 8 \%$ ) using ${ }^{201} \mathrm{Tl}$ and ${ }^{99 \mathrm{~m}} \mathrm{Tc}-\mathrm{MIBI}$ scans, comparing conventional protocols with reinjection protocols. 122 regions had permanent defects in the conventional protocol with ${ }^{201} \mathrm{Tl} ; 57$ (47\%) of them had transient defects in the reinjection protocol. Using the protocol with ${ }^{99 \mathrm{~m}} \mathrm{Tc}-\mathrm{MIBI}$, there were $22(18 \%)$ regions of transient defects. Thus, ${ }^{99 \mathrm{~m}}$ Tc-MIBI did not identify 35 (29.0\%) regions when compared with the reinjection protocol. Dilsizian et al ${ }^{27}$, using similar methodology, observed an underestimation in the protocol with ${ }^{99 \mathrm{~m}} \mathrm{Tc}-\mathrm{MIBI}$ of $36 \%$ when compared with the reinjection protocol. Reinjection imaging with ${ }^{201} \mathrm{Tl}$ identifies a greater number of viable myocardial regions when compared with ${ }^{99 \mathrm{~m}} \mathrm{Tc}-\mathrm{MIBI}$.

Clinical and experimental observations showed that, in normal tissue, soon after initial uptake, ${ }^{99 \mathrm{~m}} \mathrm{Tc}-\mathrm{MIBI}$ fixes in the myocardial cell, having minimal redistribution later. When depolarization of cell membranes and mitochondria occurs, as during ischemia, there is some difficulty in the storage and uptake of the tracer ${ }^{28,29}$. Possibly, more intense metabolic changes diminish the passive transport of ${ }^{99 \mathrm{~m}} \mathrm{Tc}-$ MIBI. On the other hand, $\mathrm{Na}+/ \mathrm{K}+\mathrm{ATPase}$ activity, an important factor in cell survival, would hardly interfere with ${ }^{201} \mathrm{Tl}$ extraction (active transport).

The statistical analysis demonstrated that the reinjection protocol was the best approach for detecting viable myocardium, and there was an important depreciation of ${ }^{99 \mathrm{~m} T c-M I B I}$ when compared with ${ }^{201} \mathrm{Tl}$ for myocardial viability identification.

Positron emission tomography ${ }^{30}(\mathrm{PET})$ is considered the most sensitive noninvasive technique for identifying viable myocardium and has been used for comparison with other methods. Several studies ${ }^{20,31}$ showed that ${ }^{201} \mathrm{Tl}$ positivity after reinjection is as sensitive as PET imaging with 18-F-deoxyglucose (FDG) in viability detection. Agreement between methods was $88 \%$ in the $1^{\text {st }}$ study and $85 \%$ in $2^{\text {nd }}$. Other studies ${ }^{19,29,32-34}$ showed similar predictive values, using recovery of wall motion observed after revascularization as the gold standard: PPV of $78 \%$ to $85 \%$ for PET and $73 \%$ to $87 \%$ for the reinjection protocol; NPV of $80 \%$ to $92 \%$ for PET and $75 \%$ to $100 \%$ for the reinjection protocol. In the present study the reinjection protocol had similar predictive values: positive of $83.3 \%$ and negative of $80.0 \%$, using perfusion recovery assessed by greater myocardial uptake after surgical revascularization.

A significant relationship was found between myocardial perfusion recovery and regional wall motion recovery $(\mathrm{p}=0.011)$ after revascularization in myocardial infarction areas.

These data are relevant to the observation that the metabolic abnormalities due to inappropriate perfusion recover after coronary flow restoration. The presence of chronic myocardial contractile dysfunction is not enough to indicate revascularization. The extent of the area potentially recoverable - viable myocardium - allied to proper coronary anatomy, could lead to change in the clinical condition of a patient after revascularization.

Probably due to the lack of other more objective and precise methods for CAD identification, coronary angiography, despite its limitations, has been used to evaluate the diagnostic precision of the methods used for studying myocardial ischemia. Angiography also has a number of problems related to the possibility of underestimation or overestimation of coronary lesions ${ }^{35}$. Quantitative coronary angiography ${ }^{36}$ seems to be more objective than visual estimation but also there are divergences among the several methods used (i.e. density against geometric method), especially when analyzing unstable and eccentric plaques. There is the possibility that the degree of anatomic stenosis does not express the real coronary flow in specific situations such as during cardiac stress ${ }^{37,38}$.

Evaluations of perfusion and LV contractile function have some limitations, especially because of the absence of a precise and of a practical quantitative method. The best quantification method currently available in practice is the semiquantitative scoring system. Therefore, the method is observer-dependant, causing intra and interobserver variability common to most diagnostic methods.

In this study, intra and interobserver variability were, respectively, $4.6 \%$ and $7 \%$ for coronary angiography; $3 \%$ and $4 \%$ for left ventriculography; $2 \%$ and $4 \%$ for the stress test; and $4 \%$ and $5.1 \%$ for the myocardial perfusion scintigraphy.

Despite these limitations, intra and interobserver variabilities were acceptable. Myocardial perfusion scintigraphy and coronary angiography with visual quantification are accepted and performed routinely in most medical centers.

\section{Acknowledgments}

To Profs. José A. Marin-Neto, Giovanni Bellotti, Adib D. Jatene and Sergio A. Oliveira, and to staff of the nuclear medicine department of the Heart Institute-HCFMUSP. 


\section{References}

1. Franciosa JA, Wilen M,Ziesche S, Cohn JN. Survival in men with severe chronic left ventricular failure due to either coronary heart disease or idiopathic dilated cardiomyopathy. Am J Cardiol 1983; 51: 831-6.

2. Primo G, Le Clerc JL, Goldstein JP, De Smet JM, Joris MP. Cardiac transplantation for the treatment of end-stage ischemic cardiomyopathy. Adv Cardiol 1988; 36 293-7.

3. Kriett JM, Kaye MP. The registry of the international society for heart transplantation: seventh official report ñ 1990. J Heart Transplant 1990; 9: 323-30.

4. Iskandrian AS, Shelbert HR. Myocardial viability assessment. J Nucl Med 1994a ; 35 (suppl): 1S-3S

5. Lucas JR, Botvinick EH, Dae MW. Myocardial viability; evidence provided by analysis of left ventricular systolic function. Coron Artery Dis 1993; 4: 485-94.

6. New York Heart Association: the criteria committee. Inc: Diseases of the heart an blood vessels. Nomenclature and criteria for 6th ed. Boston Little Brown, 1964.

7. Braunwald E. Heart Disease. A Textbook of Cardiovascular Medicine. 5th ed. Philadelphia: WR Saunders Co, 1996.

8. Tranchesi J. Eletorcardiograma Normal e PatolÛgica. NoÁies de Vectorcardiografia. 6a ed. São Paulo: Editora Athenas, 1983: 724.

9. Arie S. Circulação colateral com fator de proteção do miocárdio em portadores de insuficiência coronária crônica (Master thesis). São Paulo: Faculdade de Medicina da Universidade de São Paulo, 1977:53

10. Dodge HT, Sandler H, Bellew BW, Lord Jr JD, Seatle W. The use of biplane angiography for the measurement of left ventricular volume in man. Am Heart J 1960; 60: 762

11. Kennedy JW, Baxley WA, Figley MN. Quantitative angiography. The normal left ventricle in man. Circulation 1966, 34:272

12. Consenso Nacional de Ergometria. Florianópolis, SC. Arq Bras Cardiol 1995; 65: 189-211

13. Ellestad MA. Stress Testing. Principles and Practice. $3^{\text {rd }}$ ed. Philadelphia: Daves Company, 1986: 526.

14. Rosner B. Fundamentals of Biostatistics $-2^{\text {nd }}$ ed. Boston: PWS Publishers, 1986: 584.

15. Fleiss JL. Statistical Methods for Rates and Proportions. New York: John Wiley \& Sons, 1981: 325

16. SAS Institute Inc., SAS/STAT User's Guide, Version $6,4^{\text {th }}$ ed, vol.2, Cary, NC: SAS Institute. 1989: 1686.

17. Hochberg MS, Parsonnet V, Gielschinsky I, Hussain SM. Newark NJ. Coronary artery bypass grafting in patients with ejection fractions below forty percent. J Thorac Cardiovasc Surg 1983; 86: 519-27.

18. Coronary Artery Surgery Study (CASS): a randomized trial of coronary artery bypass surgery: survival data. Circulation 1983; 68: 939-50.

19. Dilsizan V, Rocco T, Freedman N, Martins BL, Bonow RO. Enhanced direction of ischemic but viable myocardium by the reinjection of thallium after stressredistribution imaging. N Engl J Med 1990; 323: 141-6.

20. Tamaki N, Ohtani H, Yamashita K, et al. Metabolic activity in the areas of new fillin after thallium-201 reinjection: comparison with positron emission tomography using fluorine-18-deoxyglucose. J Nucl Med 1991; 32: 673-8.

21. Hendel RC. Single photon perfusion imaging for the assessment of myocardial viability. J Nucl Med 1994; 35: 235-315.
22. Strauss HW, Harrison K, Langan JK, Lebowitz E, Pitt B. Thallium 201 for myocardial imaging: relation to thallium-201 to regional myocardial perfusion. Circulation 1975; 51: 641-5

23. Weich HF, Strauss W, Pitt B. The extraction of thallium-201 by the myocardium. Circulation 1977; 56: 188-97.

24. Pohost GM, Alpert NM, Ingwall JS, Strauss W. Thallium-201 redistribution mechanism and clinical utility. Semin Nucl Med 1980; 10: 70-93.

25. Camici P, Araujo LI, Spinks T, et al. Increase uptake of $18 \mathrm{~F}$-fluorodeoxyglucose in post-ischemic myocardium in patients with exercise induced angina. Circulation 1986; 74: 81-6.

26. Cuocolo A, Pace L, Ricciardelli B, Chiariello M, Trimarco B, Salvatore M. Identification of viable myocardium in patients with chronic coronary artery disease: comparison of thallium-201 scintigraphy with reinjection and technetium-99m methoxyisobutyl. J Nucl Med 1992; 33: 505-11.

27. Dilsizian V, Arrighi JA, Diodati JG, et al. Myocardial viability in patients with chronic coronary artery disease: comparison of Tc-99m MIBI with Thallium reinjection and F-18 fluorodeoxyglucose. Circulation 1994; 89: 578-87.

28. Okada RD, Glover D, Gaffney T, Williams S. Myocardial kinetics of technetium99m hexakis-2-methoxy-2-methypropyl-isonitrile. Circulation 1988; 77: 491-8.

29. Sinusas AJ, Watson DD, Cannon JM, Jr ME, Beller GA. Effect of ischemia and postischemic dysfunction on myocardial uptake of technetium- $99 \mathrm{~m}$ labeled methoxyisobutyl isonitrile and thallium-201. J Am Coll Cardiol 1989; 14: 1785-93.

30. Gropler RJ, Bergmann SR - Myocardial viability ñ what is the definition?J Nucl Med 1991; 32: 10-2.

31. Bonow RO, Dilsizian V, Cuocolo A, Bacharach SL. Identification of viable myocardium in patients with coronary artery disease and left ventricular dysfunction: comparison of thallium scintigraphy with reinjecton and PET imaging with 18F-fluorodeoxyglucose. Circulation 1991; 83: 26-37.

32. Tillisch J, Brunken R, Marshall R, et al. Reversibility of cardiac wall-motion abnormalities predicted by position tomography. N Engl J Med 1986; 314: 884-8.

33. Tamaki N, Yonekura Y, Yamashita K, Saji H, Magata Y, Senda M. Position emission. Position emission tomography using fluorine-18-deoxglucose in evaluation of coronary artery bypass grafting. Am J Cardiol 1989; 64: 860-5.

34. Ohtani H, Tamaki N, Yonekura Y, et al. Value of thallium-201 reinjection after delayed SPECT imaging for predicting reversible ischemia after coronary artery bypass grafting. Am J Cardiol 1990; 66: 394-9.

35. Fleming RM, Kiekeeide RL, Gould KL. Patterns in visual interpretation of coronary arteriograms as detected by quantitative coronary angiography. J. Am Coll Cardiol 1991; 18: 945-51

36. Rodrigues AS, Santaera O, Fernandez M, et al. ñ Digital coronaryangiography: a new approach in the analysis of atherosclerotic plaque. Medicine 1991; 51: 209-16.

37. Meyer Sl, Dousk GCM, Twieg DB ñ Influence of dobutamine on hemodynamics and coronary blood flow in patients with and without coronary artery disease. Am J Cardiol 1976; 38: 103-8

38. White $\mathrm{CW}$, Wright CB, Doty DB, et al. Does visual interpretation of the coronary arteriogram predict the physiologic importance of a coronary stenosis? N Engl J Med 1984; 310: 819-24. 